\title{
CLONAL DIVERSITY IN POPULATIONS OF DAPHNIA PULEX REPRODUCING BY OBLIGATE PARTHENOGENESIS
}

\author{
PAUL D. N. HEBERT AND TERI CREASE* \\ Dept. of Biology, University of Windsor, Windsor, ON N9B 3P4, Canada
}

Received 5.xi.82

\section{SUMMARY}

\begin{abstract}
Allozyme studies of Daphnia pulex populations in southern Ontario revealed marked Hardy-Weinberg deviations, complete gametic phase imbalance, and high heterozygosity values. These genotypic characteristics reflect the loss of sexual reproduction; individuals reproduce by obligate parthenogenesis. Thirtynine clones were identified in the twenty-one habitats surveyed; a few of these clones retained the ability to produce male offspring. Several clones were present in many habitats, but most of the thirty-nine clones were found in only a single pond. The large genetic distances between coexisting clones suggested that in situ mutational divergence was not the source of within habitat clonal diversity. Ecological differences between clones were suggested by distributional patterns and by temporal surveys of clone frequencies.
\end{abstract}

\section{INTRODUCTION}

It is often assumed that the shift from one breeding system to another results from a short-term fitness advantage accruing to the genotypes adopting the new breeding system. Yet there is growing evidence that the loss of sexual reproduction may often be a consequence of a class of mutations (sex-limited meiosis suppressors) whose spread through the population is difficult to stop, even if the new breeding system is disadvantageous. Richards (1973) argued that such mutations were responsible for the spread of asexuality in the plant genus Taraxacum and his model may be broadly applicable to monoecious plants. Jaenike and Selander (1979) used a related model to explain the loss of sexual reproduction in oligochaetes and their model may apply to many hermaphroditic species. Hebert $(1978,1981)$ advanced a similar explanation for the loss of sex in cladoceran crustaceans and some other cyclic parthenogens. These models all assume that a mutation arises which suppresses meiosis during female, but not during male gamete formation. As a consequence the male gametes are able to spread the gene for asexuality to female lines which produce haploid eggs. The gene for asexuality is, in a sense, contagious. The model is attractive because it provides a simple explanation for the displacement of sexual forms by their asexual derivatives. Moreover, the fact that the asexual females of many taxa continue to produce males (or male structures) indicates that the loss of sexual reproduction was not preceded or even accompanied by the loss of males. The genetic consequences of the spread of asexuality via a sex limited meiosis suppressor are clear-the asexual taxon will assimilate the genotypic diversity of the parent species. If, for U.S.A.

* Present address: Dept. of Biology, Washington University, St. Louis, Missouri 63130 
instance, the sexual parent was polymorphic for two alleles at a particular locus, one would expect three genotypes to be represented among its clonal derivatives. Assuming that the parent species was polymorphic at a typical percentage of its loci, thousands of different clones should be represented in the newly arisen clonal complex. This initial clonal diversity should be reduced through time by chance and selective extinction and be augmented by mutation.

The hypothesis that obligate asexuality in the cladocerans is the consequence of sex-limited meiosis suppressors remains untested. Indeed patterns of genotypic diversity in such asexual species have not been compared with those expected if the hypothesis were valid. To provide the data required for such comparison, a study of clonal diversity was undertaken on Daphnia pulex; a species which is perhaps the most-widespread zooplankter in freshwater habitats of the northern hemisphere. Its range extends from the sub-tropics to the high arctic and from arid habitats to coastal rainforests. The species is not restricted to the Holarctic, but is also found in South America and Africa. Although its abundance may have been exaggerated by confusion with closely related species such as $D$. curvirostris, $D$. obtusa and $D$. pulicaria, careful systematic studies have confirmed its broad distribution (Johnson, 1952; Brooks, 1957). The species is morphologically variable; some of this variation is known to be environmentally induced. For example, individuals of many $D$. pulex clones produce spikes on their dorsal carapace when exposed to a water soluble molecule released by the predaceous dipteran larva Chaoborus (Kreuger and Dodson, 1981; Schwartz and Hebert in prep). Other variation seems to be genetic in origin; in parts of its range $D$. pulex shows morphological convergence to closely related species. Brooks (1957) has argued that these morphological intermediates reflect introgressive hybridization.

Most cladoceran crustaceans reproduce by cyclic parthenogenesis. Females produce diploid parthenogenetic eggs which develop into either male or female offspring depending upon environmental conditions. Environmental factors also provoke the shift from the production of parthenogenetic eggs to haploid ephippial eggs which need to be fertilized to develop. Despite the frequency with which $D$. pulex has figured in textbook discussions of cyclic parthenogenesis, recent work has shown that populations in southwestern Ontario reproduce by obligate parthenogenesis (Hebert and Crease, 1980). Females in these populations produce diploid ephippial eggs which hatch into genetic replicates of their mother. The present study aimed at providing a more thorough analysis of the genetic characteristics of populations of $D$. pulex reproducing in this fashion.

\section{MATERIALS AND METHODS}

D. pulex was collected from two areas (Windsor, Kingston) in Ontario about $700 \mathrm{~km}$ apart. Eleven populations were studied near Windsor and ten near Kingston. All ponds were sampled in early spring, shortly after the populations were reestablished from ephippial eggs. In each area ponds were sampled in both natural (forested) and disturbed (urban-agricultural) habitats. The forest ponds contained abundant leaf litter and were shaded, while the ponds in disturbed habitats contained much less organic debris, 
and were exposed to direct sunlight. Five of the Windsor habitats (Cedar Springs, Charing Cross-1, Charing Cross-2, Bloomfield, Kingsville) were in farmland and four (Windsor $-1,-2,-3$, Cottam) were in urban settings. Seven of these nine ponds were in cleared land, but the Windsor- 2 pond was surrounded by a small stand of trees, and the basin of the Cottam pond was tree filled. The remaining two ponds in the Windsor area were located in a forest setting at Rondeau Provincial Park. Nine of the Kingston ponds were in rural habitats-some were in dense forest, others were in shallow depressions filled with trees, reminiscent of the Cottam habitat. The remaining pond was situated in a cleared area in the town of Odessa.

The ponds had surface areas ranging from $10-300 \mathrm{~m}^{2}$. Each pond was sampled by drawing a plankton net across it at several points along its longest dimension. All ponds were less than $0.5 \mathrm{~m}$ in depth, but care was taken to ensure that the entire range of depths was sampled. From 44 to 309 individuals were analyzed from the eleven Windsor populations for each of four enzymes commonly polymorphic in the area. In addition clones were established from at least 24 randomly chosen individuals from each Windsor population and from at least 48 individuals from each Kingston population. Loss of clones during establishment was neglible. Each clone was typed for its allozyme pattern at 11 loci-lactate dehydrogenase (LDH), phosphoglucomutase (PGM), phosphoglucose isomerase (PGI), tetrazolium oxidase (TO-3), malate dehydrogenase (MDH), glutamate oxaloacetate transaminase (GOT), fumarase (FUM), xanthine dehydrogenase (XDH), glucose-6-phosphate dehydrogenase (G6PDH), and amylase (two loci, AMY-1, AMY-2). The 479 clones isolated from the Kingston area, and a representative of most of the Windsor clones were additionally scored for leucine aminopeptidase, esterase, and alkaline phosphatase. Alleles were numbered in order of increasing mobility, using the same allelic designations as Hebert and McWalter (1983). Thus PGI (11) refers to an animal or clone homozygous for the " 1 " allele at the phosphoglucose isomerase locus, while (14) refers to a heterozygote for the " 1 " and " 4 " alleles, etc.

The heritability of the variation detected in allozyme patterns was established by repeated analysis of clonal lines. The genetic basis of the variation in allozyme patterns could not be investigated directly because of the completely asexual mode of reproduction. Instead the genetic basis has been inferred from the results of formal genetic studies on closely related taxa (D. magna, D. carinata, $D$. obtusa $)$ capable of sex.

Laboratory stocks of each clone were regularly checked to determine if males were present. Ephippial eggs from a number of clones were hatched, in order to determine if segregation occurred during ephippial egg production.

The data on clonal genotypes were used to calculate average heterozygosity per locus. In addition, Nei's (1975) measures of genetic divergence, $I$ and $D$, were calculated for each pair of clones. Nei's statistics require gene frequency data which in this case were calculated from the genotypes of individual clones. Single link cluster analysis was performed on the matrix of genetic distances among clones to construct a dendrogram. The clustering procedure was based on average genetic distance and used the BMD-P1M program. 


\section{REsults}

\section{(i) Enzyme phenotypes}

FUM, XDH, G6PDH, MDH, TO-3, and GOT patterns were invariant in all 21 populations. The allozyme phenotypes (either single bands or two bands in the case of $\mathrm{MDH}$ ) were compatible with the conclusion that individuals were homozygous at these loci. The genotypic designations at each locus were as follows: FUM (22), XDH (11), G6PDH (11), MDH (11), TO-3 (33), GOT (22).

The remaining enzymes were variable in both the Kingston and Windsor populations. Two LDH phenotypes were seen, a three-banded homozygote pattern (11) and a nine-banded heterozygote (13). Phenotypes at the PGI locus could be explained by assuming that two alleles were present, as two single-banded homozygote patterns $(11,44)$ and a triple-banded heterozygote pattern (14) were seen. Five phenotypes were present at both PGM and AMY-1. Homozygote patterns were single-banded, while heterozygotes had two zones of activity. The variation at these loci could be explained by assuming that three alleles were present at each; the missing phenotype in each case was the heterozygote between the two alleles with the greatest mobility difference in their products. AMY-2 phenotypes could be explained by assuming that five normal activity $(0,1,2,3,4)$ and one null activity (5) alleles were present. Again homozygotes were single- and heterozygotes double-banded.

Variation in LAP, EST and ALK phenotypes was difficult to interpret because qualitative differences were common among clones. From 3-10 bands of ALK activity were present; these differences were stable within clonal lineages indicating that the variation was heritable. In the case of ALK, clonal phenotypes were compared on the basis of pattern. It proved possible in the case of LAP and EST to ascribe the control of one zone of activity to a particular locus. Both loci apparently produced monomeric products as homozygotes were single-banded and heterozygotes doublebanded. Four alleles were present at both EST-1 and LAP-1.

\section{(ii) Genotypic frequencies in the Windsor populations}

Genotypic frequencies were analyzed in the eleven Windsor populations at four loci found to be polymorphic in the area (table 1). Large deviations from Hardy-Weinberg equilibrium were common in the populations (table 2 ). The nature of these deviations was not consistent, even within an individual population. For example the Cedar Springs population was nearly fixed for heterozygotes at LDH, but had a large deficiency of heterozygotes at PGM.

\section{(iii) Clonal diversity at Windsor}

Among the 646 individuals isolated from the Windsor habitats, 22 genetically different clones were recognized (tables 3 and 4). Based on the eleven gene loci scored for each clone, clonal heterozygosities ranged from 0-36.4 per cent with an average of 15.3 per cent. The 22 clones varied greatly in abundance. Clones W1 and W6 were the most common, being represented by 225 and 89 individuals respectively, while six clones were 


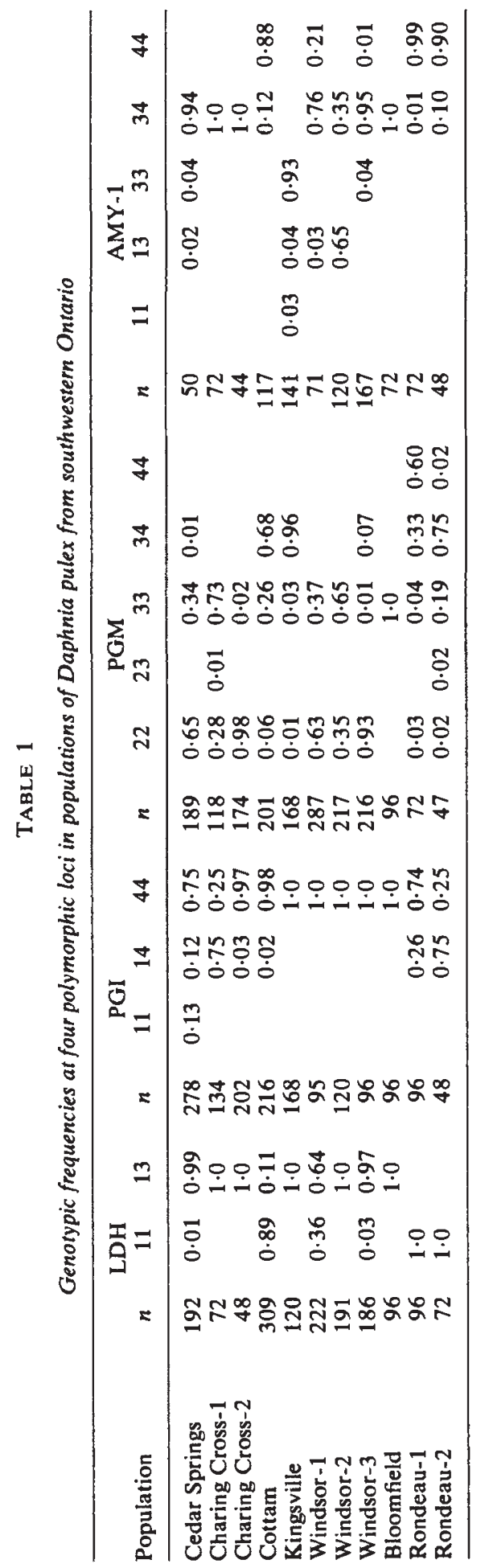


TABLE 2

Chi-square values resulting from comparison of the genotypic frequencies in table 1 with those expected assuming Hardy-Weinberg equilibrium

\begin{tabular}{|c|c|c|c|c|}
\hline \multirow[b]{2}{*}{ Population } & \multicolumn{4}{|c|}{ Locus } \\
\hline & LDH & PGI & PGM & AMY-1 \\
\hline Cedar Springs & $188 \cdot 0^{*}$ & $96 \cdot 7^{* *}$ & $183 \cdot 7^{*}$ & $41 \cdot 0^{*}$ \\
\hline Charing Cross- 1 & $72 \cdot 0^{*}$ & $47 \cdot 4^{*}$ & $113 \cdot 1^{*}$ & $72 \cdot 0^{*}$ \\
\hline Charing Cross- 2 & $48 \cdot 0^{*}$ & $0 \cdot 0$ & $1 \cdot 4$ & $44 \cdot 0^{*}$ \\
\hline Cottam & $1 \cdot 0$ & 0.0 & $42 \cdot 0^{*}$ & $0 \cdot 0$ \\
\hline Kingsville & $120 \cdot 0^{*}$ & - & $149 \cdot 3^{*}$ & $1 \cdot 2$ \\
\hline Windsor-1 & $50 \cdot 2^{*}$ & - & $287 \cdot 1^{*}$ & $26 \cdot 1^{*}$ \\
\hline Windsor-2 & $191 \cdot 0^{*}$ & - & $217 \cdot 0^{*}$ & $120 \cdot 0^{*}$ \\
\hline Windsor-3 & $167 \cdot 1^{*}$ & - & $8 \cdot 3^{* *}$ & $133 \cdot 5^{*}$ \\
\hline Bloomfield & $96 \cdot 0^{*}$ & - & - & $72 \cdot 0^{*}$ \\
\hline Rondeau-1 & - & $0 \cdot 2$ & $0 \cdot 1$ & $0 \cdot 0$ \\
\hline Rondeau-2 & - & $4 \cdot 0^{* *}$ & $17 \cdot 3^{*}$ & $0 \cdot 0$ \\
\hline
\end{tabular}

* Probability of fit to H.W.E. 0.001.

*** Probability of fit to H.W.E. $0 \cdot 05$.

TABLE 3

Genotypic characteristics of the Windsor clones. The heterozygosity estimates were calculated without including EST-1 data

\begin{tabular}{lccccccc}
\hline Clones & LDH & PGI & PGM & AMY-1 & AMY-2 & EST-1 & $\begin{array}{c}\text { Average } \\
\text { heterozygosity }\end{array}$ \\
\hline W1 & 13 & 44 & 22 & 34 & 22 & 13 & $0 \cdot 182$ \\
W2 & 13 & 44 & 33 & 34 & 22 & 13 & $0 \cdot 182$ \\
W3 & 13 & 11 & 33 & 34 & 22 & & $0 \cdot 182$ \\
W4 & 13 & 11 & 33 & 34 & 12 & 24 & $0 \cdot 273$ \\
W5 & 13 & 44 & 34 & 33 & 12 & 33 & $0 \cdot 273$ \\
W6 & 13 & 14 & 33 & 34 & 12 & 24 & $0 \cdot 364$ \\
W7 & 13 & 44 & 34 & 13 & 22 & & $0 \cdot 273$ \\
W8 & 11 & 44 & 34 & 44 & 22 & 33 & $0 \cdot 091$ \\
W9 & 11 & 44 & 33 & 44 & 22 & 33 & $0 \cdot 000$ \\
W10 & 11 & 44 & 34 & 44 & 23 & 33 & $0 \cdot 182$ \\
W11 & 13 & 44 & 34 & 33 & 23 & 33 & $0 \cdot 273$ \\
W12 & 13 & 44 & 33 & 13 & 22 & 23 & $0 \cdot 182$ \\
W13 & 11 & 44 & 33 & 44 & 55 & 13 & $0 \cdot 000$ \\
W14 & 13 & 44 & 22 & 33 & 23 & & $0 \cdot 182$ \\
W15 & 11 & 44 & 44 & 44 & 55 & 33 & $0 \cdot 000$ \\
W16 & 11 & 14 & 33 & 44 & 22 & 33 & $0 \cdot 091$ \\
W17 & 11 & 44 & 22 & 44 & 22 & 33 & $0 \cdot 000$ \\
W18 & 11 & 14 & 44 & 44 & 22 & & $0 \cdot 091$ \\
W19 & 11 & 14 & 34 & 44 & 22 & & $0 \cdot 182$ \\
W20 & 11 & 44 & 34 & 34 & 22 & & $0 \cdot 182$ \\
W21 & 11 & 44 & 23 & 34 & 22 & & $0 \cdot 182$ \\
W22 & 11 & 44 & 44 & 44 & 22 & 23 & $0 \cdot 000$ \\
\hline
\end{tabular}

represented by only a single individual. Table 4 shows that 1 to 7 clones were found in a habitat. The ponds in forest habitats (Rondeau-1, -2 , Cottam) contained significantly more clones than those in open habitats $(\bar{x}$ forest $=6 \cdot 0, \bar{x}$ open $=2 \cdot 75 ; t=2 \cdot 85,9$ d.f., $p 0 \cdot 02$ ). Clonal distributions appeared not to be random. Thus clones W1 or W6 predominated in all 


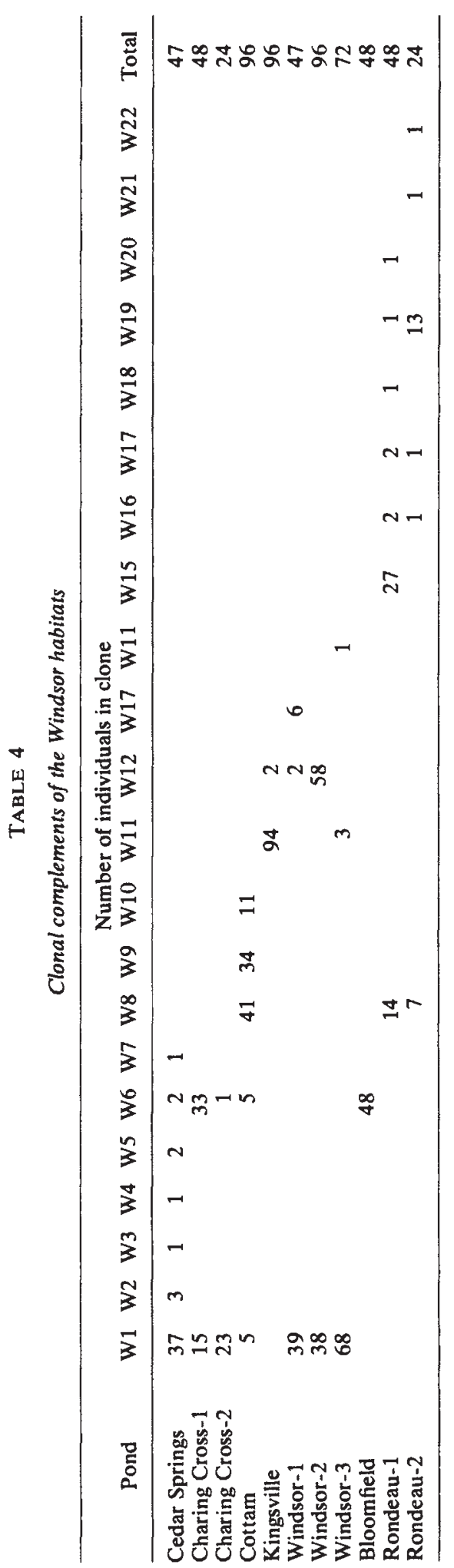


but 1 of the 8 ponds in open habitats, but were absent at Rondeau and rare at Cottam.

The clonal stocks contained the same genotypes that were detected in the survey of individuals directly from nature, indicating that a representative sample of each population had been established. These clonal stocks provided additional evidence for the restriction of sexual reproduction for they revealed not only that genotypic frequencies at individual loci deviated from Hardy-Weinberg equilibrium, but also that non-random associations existed among variants at different loci. Thus allozyme variation was noted at four loci (LDH, PGM, AMY-1, AMY-2) in the Windsor-1 population with two genotypes present at three of these loci and three genotypes at the remaining locus. Although twenty-four different multi-locus genotypes were possible, only three clones were detected (table 5). These three

TABLE 5

Genotypes detected in the Windsor-1 population

\begin{tabular}{rrrrrr}
\hline \multicolumn{5}{c}{ Clone } & \multicolumn{5}{c}{ Genotype } \\
& \multicolumn{1}{c}{$n$} & LDH & PGM & AMY-1 & AMY-2 \\
\hline 1 & 39 & 13 & 22 & 34 & 22 \\
12 & 2 & 13 & 33 & 13 & 22 \\
13 & 6 & 11 & 33 & 44 & 55 \\
\hline
\end{tabular}

genotypes (clones) were very genetically distinct. Clone 13 was most divergent being homozygous for a different AMY-2 allele from the other two clones. In addition it shared no alleles with clone 1 at the PGM locus, and only 1 allele at LDH and AMY-2. It differed from clone 12 at AMY-1 and also shared only 1 allele at LDH. Clones 1 and 12 were themselves fixed for different alleles at PGM and shared only 1 allele at AMY-1. The genotypic associations noted in the Windsor-2 population were identical to those noted at Windsor-1. The Windsor populations were not unique. Of eight potentially distinguishable genotypes in the Charing Cross populations only two were present. These two genotypes were fixed for different alleles at PGM and shared only one allele at PGI and AMY-2. Similar deviations from genotypic distributions expected in the presence of sexual reproduction were noted in most of the other populations.

The Windsor clones were maintained in the laboratory for a year before their LAP, EST and ALK phenotypes were scored. During this period a number of clones, especially those from the Rondeau habitats, died out. However, a representative of each of the remaining clones from the eleven populations was scored for these enzymes. This survey provided abundant evidence of additional genetic differences among clones, but no new clones were detected. Thus W1 clones isolated from seven different habitats were all 11 homozygotes at LAP-1 and 13 heterozygotes at EST-1. In addition to gene substitutions, qualitative differences in ALK and LAP phenotypes were evident among clones; individuals of the W12 clone from all three habitats in which it was found possessed three zones of LAP activity migrating anodally to the LAP-1 zone, while the remaining Windsor clones had only a single additional zone of LAP activity. 
Two of the Windsor populations were sampled on several occasions to determine the stability of clonal frequencies (table 6). Significant shifts in clone frequencies during an annual cycle occurred in both the Windsor-1 population in $1979\left(x_{1}^{2}=98 \cdot 6, p 0.001\right)$ and in the Charing Cross-1 population in both $1978\left(x_{1}^{2}=52 \cdot 5, p 0 \cdot 001\right)$ and $1979\left(x_{1}^{2}=47 \cdot 2, p 0 \cdot 001\right)$.

TABLE 6

Temporal survey of clone frequencies in two habitats near Windsor

\begin{tabular}{rrcc}
\hline \multirow{2}{*}{ Charing Cross-1 } & \multicolumn{2}{c}{ Proportion } \\
Date & $n$ & Clone W1 & Clone W6 \\
\hline 25.5 .78 & 60 & 0.93 & 0.07 \\
4.6 .78 & 47 & $0 \cdot 26$ & 0.74 \\
16.4 .79 & 94 & 0.20 & 0.80 \\
11.6 .79 & 96 & 0.70 & 0.30 \\
7.5 .81 & 24 & & $1 \cdot 00$ \\
12.5 .81 & 120 & 0.02 & 0.98 \\
\hline
\end{tabular}

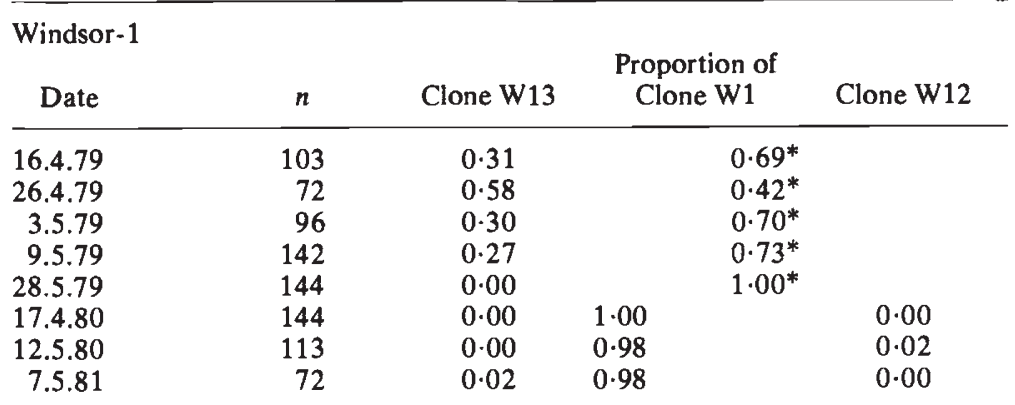

* Clones W1 and W12 not distinguished in 1979 samples.

\section{(iv) Clonal diversity at Kingston}

Variation was present at seven of the thirteen loci scored in the 479 individuals from the Kingston area (table 7). Genotypic data at these loci permitted the recognition of 17 different clones. Sixteen of these clones (K1-16) were found to have distinctive alkaline phosphatase patterns and no variation was found among those individuals assigned to a clone on the basis of the survey at other loci. K17 was the commonest clone detected in the study, and representatives of this clone isolated from ponds $3,5,8$, and most from pond 9 showed a similar ALK pattern. However, all individuals from pond 10 had a different pattern, as did 8 individuals from pond 9 . Heterozygosity values for the Kingston clones varied from $0-46 \cdot 2$ per cent, with an average heterozygosity of 14.0 per cent.

Allelic arrays were very similar at the five variable loci scored at both Kingston and Windsor. Thus the Kingston clones contained no new alleles at LDH, PGI, PGM, or AMY-1. Clones from the two areas shared the same four common alleles at AMY-2, but the Kingston clones contained two additional alleles.

The number of clones per pond varied from 1-4 in the Kingston habitats (table 8 ). Of the seventeen clones, eleven were found in only a single 
TABLE 7

Genotypic characteristics of the Kingston clones

\begin{tabular}{|c|c|c|c|c|c|c|c|c|}
\hline Clones & $\mathrm{LDH}$ & PGI & PGM & AMY-1 & AMY-2 & LAP-1 & EST-1 & $\begin{array}{c}\text { Average } \\
\text { heterozy- } \\
\text { gosity }\end{array}$ \\
\hline K1* & 11 & 44 & 33 & 44 & 33 & 22 & 23 & 0.077 \\
\hline K2 & 11 & 44 & 33 & 44 & 33 & 12 & 33 & 0.077 \\
\hline K3 & 11 & 14 & 33 & 44 & 23 & 11 & 23 & 0.231 \\
\hline K4 & 11 & 44 & 33 & 44 & 22 & 12 & 23 & $0 \cdot 154$ \\
\hline K5 & 13 & 14 & 33 & 34 & 12 & 24 & 13 & 0.462 \\
\hline K6 & 11 & 14 & 33 & 44 & 22 & 22 & 33 & 0.077 \\
\hline K $7^{*}$ & 11 & 44 & 34 & 44 & 22 & 22 & 23 & $0 \cdot 154$ \\
\hline K8 & 11 & 44 & 33 & 44 & 33 & 12 & 23 & $0 \cdot 154$ \\
\hline K9 & 11 & 44 & 34 & 44 & 11 & 22 & 33 & 0.077 \\
\hline K10 & 11 & 44 & 33 & 44 & 22 & 11 & 33 & $0 \cdot 000$ \\
\hline K11 & 11 & 44 & 23 & 44 & 02 & 22 & 33 & $0 \cdot 154$ \\
\hline K12* & 11 & 11 & 33 & 44 & 55 & 22 & 33 & 0.000 \\
\hline K13 & 11 & 44 & 34 & 44 & 24 & 13 & 22 & 0.231 \\
\hline K14* & 11 & 44 & 33 & 44 & 13 & 22 & 33 & 0.077 \\
\hline K15 & 11 & 44 & 44 & 44 & 33 & 11 & 33 & $0 \cdot 000$ \\
\hline K16 & 11 & 14 & 34 & 44 & 13 & 11 & 23 & 0.308 \\
\hline K17* & 11 & 44 & 34 & 44 & 55 & 22 & 23 & $0 \cdot 154$ \\
\hline
\end{tabular}

* Clones seen to produce males in laboratory culture.

habitat. Genotypic frequencies in habitats showed marked deviations from Hardy-Weinberg equilibrium, and linkage disequilibrium between loci was frequently complete. When diallelic polymorphisms with a frequency of the less common allele at 0.25 or greater were considered (table 9 ), it was found that genotype frequencies deviated significantly $(p<0.001)$ from Hardy-Weinberg equilibrium in all 16 cases. Each of the six polymorphic loci showed such deviations in one or more population, and the deviations were found in each population except Skycroft-2. Similar deviations from Hardy-Weinberg expectations were noted for the multiallelic polymorphisms. The absence of sexual reproduction was further confirmed by the great genetic divergence between coexisting clones. For instance, the two clones at Skycroft-2 shared no alleles at LAP-1 and AMY-2 and only one allele at PGI and EST-1.

\section{(v) Incidence of males and other evidence of obligate parthenogenesis}

Because of the marked Hardy-Weinberg deviations and the nonrandom associations of genotypes at different loci, it was suspected that both the Kingston and Windsor clones reproduced by obligate parthenogenesis.

Field surveys confirmed the absence of males during periods of ephippial egg production in populations near Windsor. However, during the course of extensive laboratory studies male production was observed in clone W4. Near Kingston, males were encountered fairly commonly in several of the forest habitats. In the laboratory, five clones were found to produce males (table 7). Clone K17 was common in the Crosby, Skycroft-1, and Westbrook ponds and laboratory cultures of this clone from each pond produced males. 


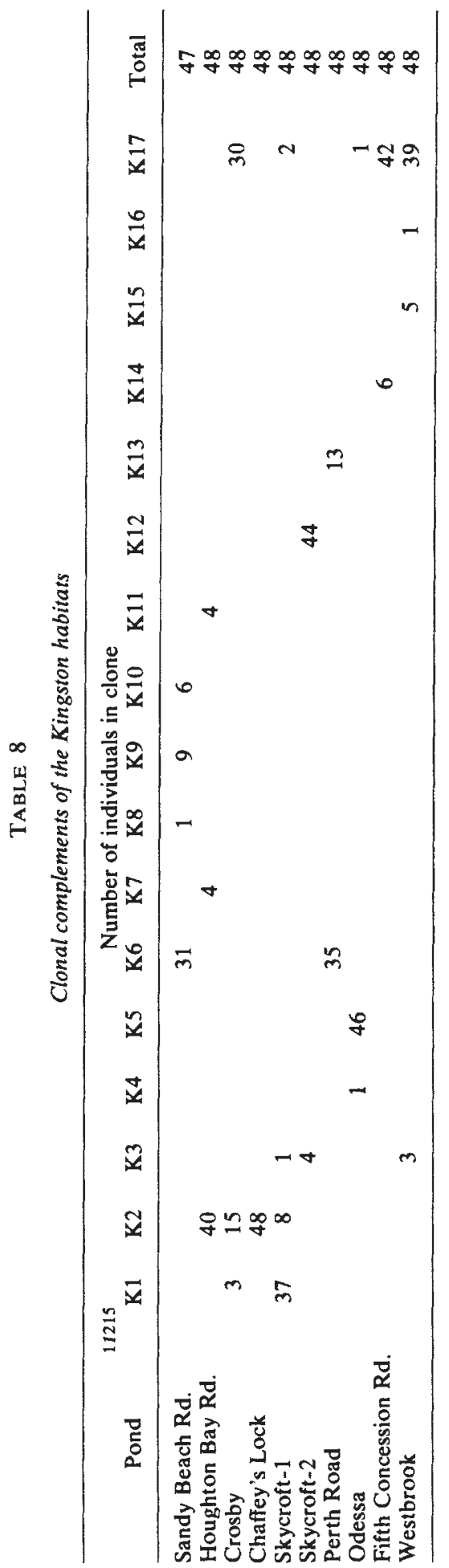


TABLE 9

Deviations from Hardy-Weinberg equilibrium in the Kingston populations. Analysis has been restricted to diallelic polymorphisms in which the frequency of the less common allele exceeded 0.25

\begin{tabular}{lcl}
\hline \multicolumn{1}{r}{ Population } & $\chi^{2}$ Value & Locus \\
\hline Houghton Bay & $24 \cdot 5$ & LAP-1 \\
Sandy Beach Rd. & $11 \cdot 4$ & PGI \\
Crosby & $9 \cdot 9$ & PGM \\
& $48 \cdot 0$ & AMY-2 \\
Chaffey's Lock & $13 \cdot 2$ & EST-1 \\
Skycroft-1 & $48 \cdot 0$ & LAP-1 \\
Perth Rd. & $24 \cdot 5$ & EST-1 \\
& $15 \cdot 8$ & PGI \\
Odessa & $48 \cdot 0$ & EST-1 \\
& $40 \cdot 6$ & LDH \\
Fifth Concession Rd. & $40 \cdot 6$ & PGI \\
& $40 \cdot 6$ & AMY-1 \\
Westbrook & $29 \cdot 0$ & PGM \\
& $29 \cdot 0$ & EST-1 \\
& 21.5 & PGM \\
\end{tabular}

It is unlikely that these lists are comprehensive-other clones probably retained the ability to produce males.

Despite the presence of males no evidence of sexual reproduction was obtained. When ephippia were collected from mass cultures of several Windsor clones no segregation was seen among 41 ephippial hatchlings scored for LDH and 58 scored for PGI. Without exception the offspring of heterozygous mothers were themselves heterozygous. More detailed studies were carried out on the Windsor (W4) clone and on three of the Kingston clones (K12, K14, K17) known to produce males. In each case, females were found to release viable ephippial eggs when isolated from males indicating that fertilization was not required to stimulate development of a diploid egg. Moreover, when 30 ephippial hatchlings were examined from each of clones $\mathrm{W} 4, \mathrm{~K} 14$, and $\mathrm{K} 17$, no segregation was seen at loci heterozygous in the present strain. K12 could not be tested because it was monomorphic at each of the loci scored.

\section{(vi) Genetic relationships among the clones}

Genetic distances among the 22 Windsor clones ranged from 0.02 to 0.40 with a mean of $0 \cdot 145$. The Kingston clones showed similar differentiation with a range from 0.02 to 0.38 and a mean distance of 0.13 . The dendrogram in fig. 1 was constructed on the basis of gene frequencies at the 11 loci which were scored in all 39 clones. Three major clusters are recognizable; the first includes 3 clones from the Windsor area, two (W1, W14) from urban habitats and one (W17) from Rondeau. All three clones were fixed for a rare homozygote (22) of PGM. Inspection of the data suggested that W17 would not have remained in this cluster if more loci had been considered. It was homozygous at both LDH and AMY-1, while the other two clones were heterozygous. EST-1 data indicated as well that 


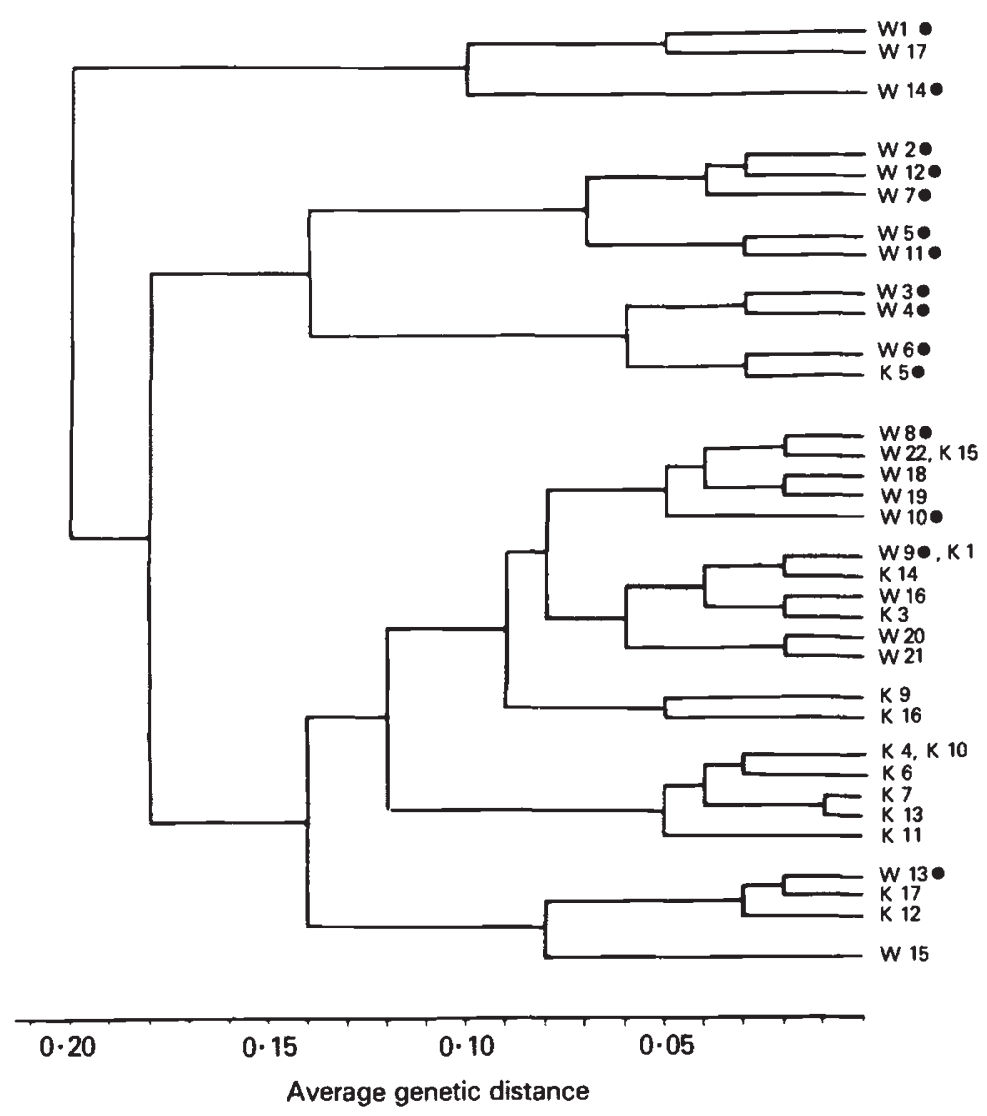

FIG. 1. Single link dendrogram showing the genetic relationships among the Kingston and Windsor clones of $D$. pulex. -clones from ponds in disturbed settings.

it was homozygous for an allele (22) common in clones belonging to cluster three, but not present in clone W1. The second cluster included nine clones (W2-K5), eight from the Windsor area and one from Kingston. All nine clones were heterozygous at $\mathrm{LDH}$ and all were found in disturbed habitats. The remaining cluster included twenty-seven clones. All were homozygous at $\mathrm{LDH}$ and most were also homozygous at AMY-1. Most of these clones were collected in forest habitats near Kingston and Windsor. Three of the Windsor area clones (W9, W10, W13) came from disturbed habitats, but W9 and W10 were from the tree filled pond at Cottam. W13 was a rare clone at Windsor -1 . Table 10 shows that the clones were not randomly distributed between habitats $\left(x_{2}^{2}=1039 \cdot 3, p 0.001\right)$; clones belonging to clusters 1 and 2 occur in open habitats, while those in cluster 3 occur in forest habitats.

\section{Discussion}

Genetic studies on cladoceran populations reproducing by cyclic parthenogenesis have shown that genotype frequencies approximate Hardy-Weinberg expectations and that variation at different loci 
TABLE 10

Number of individuals belonging to each of the three clonal clusters in two habitat types

\begin{tabular}{ccc}
\hline Cluster & $\begin{array}{c}\text { Habitat type } \\
\text { Open }\end{array}$ & Forest \\
\hline 1 & 221 & 8 \\
2 & 297 & 5 \\
3 & 9 & 585 \\
\hline
\end{tabular}

approaches linkage equilibrium for at least several generations after populations are reestablished from ephippial eggs (Hebert, 1974b; Hebert and Moran, 1981; Hann and Hebert, 1982). When populations are able to reproduce asexually for several years, Hardy-Weinberg deviations and non-random associations of genotypes develop (Hebert 1974a, $c$; Young $1979 a, b)$. However, the deviations at single loci are ordinarily due to a heterozygote excess and the linkage associations among genotypes at different loci are rarely complete. Viewed from the perspective of these earlier studies, the genetic characteristics of $D$. pulex populations in eastern and southwestern Ontario are unusual. Genotypic frequencies in most populations showed marked deviations from Hardy-Weinberg expectations. There was no consistent pattern to these deviations-at some loci heterozygotes were fixed, while at other loci heterozygotes were absent. In addition, complete linkage disequilibrium among loci was commonly observed. The unusual genetic characteristics of these populations cannot be attributed to a suppression of ephippial input. There was no evidence that populations overwintered in a motile diapause. Indeed all of the Kingston ponds and all but two of the Windsor ponds were so shallow that they froze to the bottom. Moreover, early spring samples contained only juveniles, a trait typical of populations which have been reestablished from ephippial eggs. The genotypic characteristics of these $D$. pulex populations cannot be explained by the suppression of ephippial egg hatching, but rather by the suppression of meiosis during ephippial egg formation.

The absence of sexual reproduction in these populations was supported by the rarity of males during periods of ephippial egg production. Indeed, males of several common clones (e.g. W1) were never observed. However, some clones did continue to produce males. The retention of apparently functionless males is not unique to $D$. pulex; males have been noted in other cladoceran taxa reproducing by obligate parthenogenesis (Tash, 1964; Hebert, 1981). Similarly, other asexual taxa retain structures which are only of importance during sexual reproduction (Jaenike and Selander, 1979). The reduction in clonal fitness due to retention of males may be small, because they are produced infrequently and under conditions in which the survival of female offspring is uncertain (Hebert, 1982). It may be premature to regard the males as functionless; they might fertilize the diploid eggs of competing clones resulting in non-viable triploid offspring. Detailed study of three male producing clones failed to provide any evidence of their involvement in ephippial egg production. Gynogenesis seems not to be involved. Ephippial offspring showed no segregation at variable loci and viable ephippial eggs were released in the absence of males. 
Clones of $D$. pulex reproducing by obligate parthenogenesis are not restricted to Ontario. Allozyme studies have shown that populations throughout arctic Canada reproduce in the same fashion (Hebert and McWalter, 1983; Loaring, 1982). Similarly, the genetic data on $D$. pulex in the eastern U.S.A. (Berger and Sutherland, 1978) are most readily explained by assuming that these populations also do not reproduce sexually. Lynch (1983) has reported the presence of $D$. pulex reproducing by both cyclic and obligate parthenogenesis in Illinois. He identified two groups of clones reproducing by cyclical parthenogenesis, but morphological study has revealed that one of these is $D$. obtusa and that the other is unlike $D$. pulex (pers. obs.). Thus, there is no strong evidence for sexual populations of $D$. pulex in North America. The extent to which closely related species such as $D$. schodleri, $D$. middendorffian $a$ and $D$. rosea share the loss of sexual reproduction merits analysis. It is known from cytological (Zaffagnini and Sabelli, 1972) and allozyme (Hebert and McWalter, 1983) studies that not only do populations of $D$. middendorffiana reproduce apomictically, but also that the "species" shows a close genetic relationship to $D$. pulex. This observation suggests that the taxonomic uncertainties regarding $D$. pulex and its close relatives may result not from introgressive hybridization, but rather as a consequence of attempts to impose species boundaries on an apomictic complex.

Most of the Ontario habitats contained several clones of $D$. pulex. Genetic differences among these clones were frequently too substantial to have arisen via in situ mutational divergence. Rather it seems that most habitats have been colonized by several clones. The large temporal shifts in clone frequencies noted in two habitats suggested that fitness differences existed among clones and laboratory studies have confirmed this conclusion (Loaring and Hebert, 1981). Substantial genetic differences were noted between clones found in ponds of forested versus cleared habitats. As the same genetic differences were evident in both Kingston and Windsor, chance effects are an unlikely explanation. It would seem, instead, that certain clones are adapted to forest and others to open habitats.

The extent of genetic diversity in the Ontario populations of $D$. pulex conflicts with the conclusions of those theoreticians who hold that apomictic taxa are genetically rather invariant (Maynard Smith, 1978). The species consists of not one, but hundreds or more likely thousands of different clones. While allozyme studies have revealed genotypic variation in other asexual taxa (Suomalainen and Saura, 1973; Vepsalainen and Jarvinen, 1979; Vrijenhoek, 1979; Mitter et al., 1979) D. pulex does show unusually great clonal diversity. This diversity supports the hypothesis that asexuality resulted from the spread of a sex-limited meiosis suppressor. Moreover the genotypic characteristics of the clones resemble those expected if the genetic diversity of a sexual parent species were assimilated. The clonal genotypes correspond with those anticipated in a polymorphic sexual population and allelic diversity is restrained. There is, however, one indication of differentiation after the loss of sex; clones of $D$. pulex have an average heterozygosity of 15 per cent, while individual heterozygosities in cladoceran species reproducing by cyclic parthenogenesis average only 3 per cent (Hebert and McWalter, 1983). If one assumes that its asexual ancestor was not unusually polymorphic, then clonal heterozygosities must have been increased by mutation or by the greater extinction of clones 
with low heterozygosity. Based on a mutation rate to new allozyme variants of $10^{-6} /$ gene copy/generation, approximately 60,000 generations would be required to bring about a 12 per cent increase in heterozygosity $\left(10^{-6} \times\right.$ $6 \times 10^{4}=0 \cdot 12$ ). Thus it seems unlikely that these asexual clones have persisted for much more than 20,000 years. Indeed, if the differential extinction of clones is responsible, at least in part, for their increased heterozygosity, then their origin may be much more recent.

The present study has provided data compatible with the suggestion that asexuality in Daphnia is a consequence of the spread of a sex-limited meiosis suppressor. The study has furthermore shown that some clones reproducing by obligate parthenogenesis retain the ability to produce male offspring. It seems likely that these males offer the best means of further investigating the transition from cyclic to obligate parthenogenesis. Indeed if the males produce sperm of normal ploidy, it should be possible to directly study the genetic control of meiosis by crossing them to sexually reproducing conspecifics.

Acknowledgements. We wish to thank J. Loaring for providing technical assistance throughout the study and Drs B. Hann, S. Schwartz, and the reviewers for their valuable criticism of the manuscript. The research was supported by a grant from the Natural Sciences and Engineering Research Council of Canada to PDNH.

\section{REFERENCES}

BERGER, E. AND SUTHERLAND, J. 1978. Allozyme variation in two natural populations of Daphnia pulex. Heredity, 41, 13-23.

BROOKS, J. L. 1957. The systematics of North American Daphnia. Mem. Conn. Acad. Arts. Sci., 13, 1-180.

HANN, B. J. AND HEBERT, P. D. N. 1982. Re-interpretation of genetic variation in Simocephalus (Cladocera, Daphniidae). Genetics, 102, 101-107.

HEBERT, P. D. N. 1974a. Enzyme variability in natural populations of Daphnia magna. II Genotypic frequencies in permanent populations. Genetics, 77, 323-334.

HEBERT, P. D. N. 1974b. Enzyme variability in natural populations of Daphnia magna. III Genotypic frequencies in intermittent populations. Genetics, 77, 335-341.

HEBERT, P. D. N. 1974c. Ecological differences between genotypes in a natural population of Daphnia magna. Heredity, 33, 327-337.

HEBERT, P. D. N. 1978. The population biology of Daphnia (Crustacea, Daphniidae). Biol. Rev., 58, 387-426.

HEBERT, P. D. N. 1981. Obligate asexuality in Daphnia. Amer. Nat., 117, 784-789.

HEBERT, P. D. N. 1983. Clonal diversity in cladoceran populations, in Population Biology: Retrospect and Prospect, Ed. C. E. King and P. S. Dawson. Columbia University Press.

HEBERT, P. D. N. AND CREASE, T. J. 1980. Clonal coexistence in Daphnia pulex (Leydig): another planktonic paradox. Science, 207, 1363-1365.

HEBERT, P. D. N. AND MORAN, C. 1981. Enzyme variability in natural populations of Daphnia carinata King. Heredity, 45, 313-321.

HEBERT, P. D. N. AND MCWALTER, D. B. 1983. Genetic variation in arctic Daphnia reproducing by obligate parthenogenesis. Evolution: in press.

JAENIKE, J. AND SELANDER, R. K. 1979. Evolution and ecology of parthenogenesis in earthworms. Amer. Zool. 19, 729-737.

Johnson, D. S. 1952. The British species of the genus Daphnia (Crustacea, Cladocera). Prod. Zool. Soc. Lond., 122, 435-462.

KREUGER, D. A. AND DODSON, S. I. 1981. Embryological induction and predation ecology in Daphnia pulex. Limnol. Oceanog., 26, 219-223.

LOARING, J. M. 1982. The ecological genetics of Daphnia. M.Sc. thesis, University of Windsor.

LOARING, J. M. AND HEBERT, P. D. N. 1981. Ecological differences among clones of Daphnia pulex. Oecologia, 51, 162-168. 
LYNCH, M. 1983. Ecological genetics of Daphnia pulex. Evolution, 37, 358-374.

MAYNARD SMITH, J. 1978. The Evolution of Sex. Cambridge University Press, Cambridge. MITTER, C., FUTUYMA, D. J., SCHNEIDER, J. C. AND HARE, J. D. 1979. Genetic variation and host plant relations in a parthenogenetic moth. Evolution, 33, 777-790.

NEI, M. 1975. Molecular Population Genetics and Evolution. North-Holland, New York.

RICHARDS, A. J. 1973. The origin of the Taraxacum agamo-species. Bot.J. Linn. Soc., 66, 189-211.

SUOMALAINEN, E. AND SAURA, A. 1973. Genetic polymorphism and evolution in parthenogenetic animals. I. Polyploid Curculionidae. Genetics, 74, 489-508.

TASH, J. C. 1964. The zooplankton of fresh and brackish waters of the Cape Thompson area, Alaska. Ph.D. diss. University of Kansas.

VEPSALAINEN, K. AND JARVINEN, O. 1979. Apomictic parthenogenesis and the pattern of the environment. Amer. Zool., 19, 739-751.

VRIJENHOEK, R. C. 1979. Factors affecting clonal diversity and coexistence. Amer. Zool., $19,787-797$.

YOUNG, J. P. W. 1979a. Enzyme polymorphism and cyclic parthenogenesis in Daphnia magna. I. Selection and clonal diversity. Genetics, 92, 953-970.

YOUNG, J. P. W. 1979b. Enzyme polymorphism and cyclic parthenogenesis in Daphnia magna. II. Heterosis following sexual reproduction. Genetics, 92, 971-982.

ZAFFAGNINI, F. AND SABELLI, B. 1972. Karyological observations on the maturation of the summer and winter eggs of Daphnia pulex and Daphnia middendorffiana. Chromosoma, $36,193-203$. 